\title{
EFEITO DO PESO DE ABATE NOS RENDIMENTOS DO PROCESSAMENTO DA PIRACANJUBA (Brycon orbignyanus, VALENCIENNES, 1849)
}

\author{
Effect of body weight on processing yields of piracanjuba (Brycon orbignyanus Valenciennes, 1849) \\ Thiago Archangelo Freato ${ }^{1}$, Rilke Tadeu Fonseca de Freitas ${ }^{2}$, Vander Bruno dos Santos ${ }^{3}$, \\ Priscila Vieira Rosa Logato ${ }^{2}$, Ana Tereza de Mendonça Viveiros ${ }^{2}$
}

\begin{abstract}
RESUMO
Objetivou-se, com o presente trabalho, avaliar o efeito do peso de abate sobre os rendimentos do processamento do peixe teleósteo piracanjuba (Brycon orbignyanus, Valenciennes, 1849). Inicialmente, alevinos com peso médio de 12 g, oriundos da Estação Experimental da Usina Hidrelétrica de Itutinga, foram estocados e cultivados em um viveiro de terra na Estação de Piscicultura do Departamento de Zootecnia da Universidade Federal de Lavras. Ao final de 18 meses de cultivo, 121 peixes foram insensibilizados, abatidos, pesados e dissecados, para a determinação das porcentagens de cabeça (\%CAB), nadadeiras (\%NAD), vísceras (\%VIS), pele com escamas (\%PE), e resíduos da filetação (\%RF) e dos rendimentos de carcaça (RCAR) e de filé sem pele (RFSP), em relação ao peso de abate. Para as análises de variância, os dados obtidos foram agrupados em 4 classes de peso $\left(\mathrm{CP}_{1}=515 \mathrm{~g}\right.$ a $629 \mathrm{~g} ; \mathrm{CP}_{2}=630 \mathrm{~g}$ a $744 \mathrm{~g} ; \mathrm{CP}_{3}=745 \mathrm{~g}$ a $859 \mathrm{~g}$; e $\mathrm{CP}_{4}=860 \mathrm{~g}$ a $\left.975 \mathrm{~g}\right)$ e as equações de regressão estimadas em função do peso médio de cada classe. Não foi constatado $(\mathrm{P}>0,05)$ efeito de classe de peso sobre RCAR, \%NAD e \%PE. Por outro lado, observaram-se efeito linear do peso de abate sobre \%CAB ( $\mathrm{P}<0,01)$, \%RF $(\mathrm{P}<0,01)$ e $\mathrm{RFSP}(\mathrm{P}<0,09)$, e efeito quadrático sobre \%VIS $(\mathrm{P}<0,05)$. As variáveis \%CAB e \%RF diminuíram, ao passo que o RFSP e \%VIS aumentaram, com o aumento do peso de abate. Pode-se concluir que, nas condições em que o experimento foi realizado, piracanjubas com maiores pesos de abate proporcionam maior RFSP e menores \%CAB e \%RF. Entretanto, a redução da \%CAB não foi suficiente para aumentar o RCAR como seria esperado, em razão provavelmente de um maior aumento da \%VIS.
\end{abstract}

Termos para indexação: Peixe, Piracanjuba, Rendimento de filé, Rendimento de carcaça.

\begin{abstract}
The objective of this study was to evaluate the effect of body weight on processing yields of a teleost fish piracanjuba (Brycon orbignyanus, Valenciennes, 1849). Firstly, fingerlings were reared in ponds in the Fish Culture of the Animal Sciences Department, Federal University of Lavras, M.G. - Brazil, for 18 months. Then, 121 fishes were insensibilized, slaughtered, weighed and dissected, in order to determine the percentages of head (\%CAB), fins (\%NAD), visceras (\%VIS), skin with scales (\%PE), and fillet residues (\%RF), and the carcass yield (RCAR) and fillet without skin yield (RFSP), in relation to body weight. For analyses of variances, data were distributed over four weight classes $\left(\mathrm{CP}_{1}=515\right.$ to $629 \mathrm{~g} ; \mathrm{CP}_{2}=630$ to $744 \mathrm{~g} ; \mathrm{CP}_{3}=745$ to 859g; and $\mathrm{CP}_{4}=860$ to $975 \mathrm{~g}$ ) and the regression equations were estimated in function of mean weight of each class. There was no effect $(\mathrm{P}>0,05)$ of weight class on RCAR, \%NAD and \%PE. However, a linear effect of body weight on \%CAB $(\mathrm{P}<0,01)$, \%RF $(\mathrm{P}<0,01)$ and RFSP $(\mathrm{P}<0,09)$ and a quadratic effect on \%VIS $(\mathrm{P}<0,05)$ were observed. The \%CAB and \%RF decreased while RFSP and \%VIS increased, with the increase of body weight. Based on these results, it can be concluded that heavier piracanjubas provide larger RFSP, smaller \%CAB and smaller \%RF. The smaller \%CAB on heavier fish did not increase RCAR, as expected probably because of the increase on \%VIS.
\end{abstract}

Index terms: Fish, Piracanjuba, Carcass yield, Fillet yield.

(Recebido para publicação em 22 de setembro de 2004 e aprovado em 7 de março de 2005)

\section{INTRODUÇÃO}

A piracanjuba (B. orbignyanus) é uma espécie de peixe de piracema, originária da Bacia do Prata, de hábito alimentar onívoro, aceitando bem dietas artificiais e apresentando rápido crescimento e facilidade de cultivo (VAZ et al., 2000). Além disso, possui carne de excelente qualidade e com boa aceitação no mercado, apresentando comportamento agressivo quando fisgado, o que torna sua criação em cativeiro uma atividade de grande interesse comercial tanto para a produção de carne como para a pesca esportiva.

Feiden \& Hayashi (1999), estudando o desenvolvimento da piracanjuba em tanques submetidos a diferentes adubações orgânicas, constataram que os

\footnotetext{
1. Aluno do curso de Mestrado em Zootecnia - Departamento de Zootecnia - Universidade Federal de Lavras/UFLA - Caixa Postal 3037 37200-000 - Lavras, MG.

2. Professor Adjunto do Departamento de Zootecnia - Universidade Federal de Lavras/UFLA.

3. Aluno do curso de Doutorado em Zootecnia - Departamento de Zootecnia - Universidade Federal de Lavras/UFLA.
} 
alevinos dessa espécie apresentam desenvolvimento uniforme, alta taxa de sobrevivência, boa conversão alimentar e rápido crescimento, o que a torna um peixe bastante promissor.

A piracanjuba é uma das espécies da fauna brasileira ameaçadas de extinção (COPAM, 1996), decorrente da construção de um grande número de barragens hidrelétricas, que impedem sua migração reprodutiva, do desmatamento ciliar, da deterioração da qualidade da água provocada pela poluição industrial e urbana, e da pesca predatória. Dessa forma, estudos mais detalhados sobre as características zootécnicas e de rendimento do processamento dessa espécie poderiam aumentar seu valor econômico e, conseqüentemente, incentivar sua criação comercial e preservação.

Para a indústria, a qualidade da carcaça do pescado é fator imprescindível para definição dos processos de preparação dos produtos e dos tipos de cortes. O rendimento de filé, além da eficiência das máquinas filetadoras ou da destreza manual do operário, depende de algumas características intrínsecas à matéria-prima, ou seja, da forma anatômica do corpo, do tamanho da cabeça e dos pesos dos resíduos (vísceras, pele e nadadeiras) (CONTRERAS-GUZMÁN， 1994; EYO, 1993; RIBEIRO et al., 1998).

De acordo com Contreras-Guzmán (1994), a forma do corpo tem grande importância no processamento do pescado pela indústria, influenciando nas operações de decapitação, evisceração e, inclusive, no rendimento da carne quando processada na forma de corpo limpo, postas ou filés. Segundo esse autor, os peixes fusiformes, como o curimbatá (Prochilodus scrofa, Steindachner, 1881) e a piracanjuba (B. orbignyanus), apresentam rendimentos relativamente altos devido à massa muscular cilíndrica, superando valores de $54 \%$ de rendimento de filé com pele. Entretanto, peixes comprimidos dos lados, como a tilápia-do-nilo (Oreochromis niloticus, Linnaeus, 1757), estão entre as espécies de menores rendimentos (rendimentos de filé com pele inferiores a 42\%).

O rendimento de filé pode variar entre as espécies e dentro da mesma espécie, possivelmente devido à falta de um sistema padrão nas metodologias de pesquisa nessa área (GASPARINO et al., 2002).

Em várias pesquisas, tem sido demonstrada a influência do peso de abate sobre o rendimento de carcaça e de filé em tilápias do Nilo (CONTRERASGUZMÁN, 1994; MACEDO-VIEGAS et al., 1997; SOUZA et al., 1998). Entretanto, poucos estudos têm sido realizados em espécies do gênero Brycon.
A determinação dos rendimentos do processamento de uma espécie e de suas relações com o peso de abate permite caracterizar o produto final e avaliar o seu potencial para a industrialização, e estabelecer o peso ideal de abate e as equações de predição dos rendimentos de carcaça e filé.

Objetivou-se com este trabalho avaliar o efeito da classe de peso de abate sobre os rendimentos do processamento da piracanjuba (B. orbignyanus).

\section{MATERIAL E MÉTODOS}

Para o presente estudo, alevinos de piracanjuba (B. orbignyanus) oriundos da Estação Experimental da Usina Hidrelétrica de Itutinga, região sul de Minas Gerais, pesando em média $12 \mathrm{~g}$, foram estocados e cultivados em um viveiro de terra de $500 \mathrm{~m}^{2}$, localizado na Estação de Piscicultura da Universidade Federal de Lavras. Os peixes foram alimentados com ração comercial, contendo os níveis de proteína bruta requeridos para cada fase do desenvolvimento, sendo a quantidade fornecida de acordo com a biomassa dos tanques.

Ao final de um período de 18 meses de cultivo e após jejum de 24 horas, 121 peixes foram despescados, insensibilizados por choque térmico, abatidos por anóxia, pesados e dissecados.

Para a obtenção dos rendimentos do processamento, as seguintes partes componentes do corpo foram pesadas: cabeça, seccionada do corpo na altura da junção com a altura vertebral (incluindo as brânquias); nadadeiras, sendo a nadadeira caudal seccionada à altura do perímetro peduncular; vísceras, compreendendo todo o conteúdo da cavidade celomática, inclusive as gônadas e a gordura celomática; carcaça (peixe eviscerado, sem cabeça e nadadeiras); pele e escamas; filé; e resíduo da filetação. A seguir, foram determinados os seguintes rendimentos e porcentagens em relação ao peso de abate:

- $\quad$ porcentagem de nadadeiras (\%NAD);

- $\quad$ porcentagem de cabeça (\%CAB);

- $\quad$ porcentagem de vísceras (\%VIS);

- rendimento de carcaça (RCAR);

- $\quad$ porcentagem de pele e escamas (\%PE);

- $\quad$ rendimento de filé sem pele (RFSP);

- $\quad$ porcentagem de resíduos da filetação (\%RF).

As classes de pesos analisadas foram:

$\mathrm{CP}_{1}$ - Peso de abate maior ou igual a 515 e menor que 630; 
$\mathrm{CP}_{2}$ - Peso de abate maior ou igual a 630 e menor que 745;

$\mathrm{CP}_{3}$ - Peso de abate maior ou igual a 745 e menor que 860;

$\mathrm{CP}_{4}$ - Peso de abate maior ou igual a 860 e menor que 975 .

As análises de variância dos dados foram realizadas utilizando-se o procedimento ANOVAG do pacote computacional SAEG - Sistema para Análises Estatísticas, versão 5.0 (UFV, 1992), decompondo o efeito do peso de abate em componentes de regressão linear, sendo escolhida a equação de regressão que melhor se ajustou aos dados.

O modelo estatístico usado nas análises de variância foi o seguinte:

$$
y_{i j}=\mu+P_{i}+e_{i j}
$$

Em que:

$\mathrm{y}_{\mathrm{ij}}=$ observação $\mathrm{j}$ na classe de peso $\mathrm{i}$;

$\mu$ = média geral;

$\mathrm{P}_{\mathrm{i}}=$ efeito da classe de peso $\mathrm{i}, \mathrm{i}=1,2,3,4$;

$\mathrm{e}_{\mathrm{ij}}=$ erro aleatório associado a cada observação.

\section{RESULTADOS E DISCUSSÃO}

Na Tabela 1 verificam-se as médias aritméticas e os coeficientes de variação dos rendimentos do processamento em cada classe de peso estudada, demonstrando o efeito do peso de abate sobre essas variáveis, com suas respectivas significâncias, de acordo com a análise de variância. Os coeficientes de variação foram baixos para todas as variáveis nas diferentes classes de peso, indicando boa precisão experimental.

De acordo com a análise de variância, não foi constatado $(\mathrm{P}>0,05)$ efeito de classe de peso sobre RCAR, \%NAD e \%PE. Por outro lado, observou-se efeito linear do peso de abate sobre \%CAB $(\mathrm{P}<0,01)$, \%RF $(\mathrm{P}<0,01)$ e RFSP $(\mathrm{P}<0,09)$, e efeito quadrático sobre \%VIS $(\mathrm{P}<0,05)$.

As porcentagens de cabeça (\%CAB) e de resíduo da filetação (\%RF) diminuíram com o aumento da classe de peso de abate (Figura 1 e 2, respectivamente), ao passo que o rendimento do filé sem pele (RFSP) aumentou (Figura 3). ContrerasGusmán (1994) afirma que existe uma relação inversa entre o tamanho da cabeça e os rendimentos de carcaça e de filé.

Pela Figura 4 observa-se que a porcentagem de vísceras (\%VIS) aumentou, em função do aumento da classe de peso, até atingir o máximo de 12,12\%, em peixes com peso médio de abate de 915,8 g. Esse aumento se deve, provavelmente, à proximidade da maturidade sexual, uma vez que foi considerado como vísceras todo o conteúdo da cavidade celomática, incluindo as gônadas e gordura celomática.

TABELA 1 - Médias e coeficientes de variação (CV) dos rendimentos do processamento nas diferentes classes de peso.

\begin{tabular}{|c|c|c|c|c|c|c|c|c|}
\hline \multirow{2}{*}{ Variáveis } & \multicolumn{2}{|c|}{ CLASSE $1(n=17)$} & \multicolumn{2}{|c|}{ CLASSE 2(n = 39) } & \multicolumn{2}{|c|}{ CLASSE $3(n=47)$} & \multicolumn{2}{|c|}{ CLASSE $4(n=18)$} \\
\hline & média & & $\mathbf{C V}$ & & médi & & $\mathbf{C V}$ & \\
\hline$P(g)$ & 577,24 & 6,24 & 692,79 & 5,04 & 797,32 & 3,56 & 901,06 & 3,49 \\
\hline$\% \mathrm{NAD}$ & 5,69 & 15,64 & 5,81 & 18,59 & 5,57 & 18,31 & 5,48 & 18,07 \\
\hline$\% \mathrm{CAB}^{1}$ & 14,57 & 6,73 & 13,41 & 10,37 & 13,33 & 7,73 & 12,81 & 9,37 \\
\hline$\% \mathrm{VIS}^{2}$ & 10,06 & 12,52 & 11,18 & 9,66 & 12,03 & 9,73 & 12,19 & 8,94 \\
\hline RCAR (\%) & 69,68 & 1,94 & 69,59 & 3,25 & 69,07 & 2,62 & 69,52 & 3,31 \\
\hline$\% \mathrm{PE}$ & 8,35 & 14,37 & 8,25 & 12,97 & 8,45 & 21,42 & 8,25 & 17,09 \\
\hline $\operatorname{RFSP}(\%)^{3}$ & 43,45 & 5,52 & 44,04 & 4,38 & 44,23 & 6,69 & 44,97 & 5,87 \\
\hline$\% \mathrm{RF}^{1}$ & 17,88 & 12,53 & 17,31 & 10,69 & 16,39 & 12,51 & 16,30 & 10,31 \\
\hline
\end{tabular}

(1) - efeito linear $(\mathrm{P}<0,01)$

(2) - efeito quadrático $(\mathrm{P}<0,05)$

(3) - efeito linear $(\mathrm{P}<0,09)$ 
Os coeficientes de determinação $\left(\mathrm{R}^{2}\right)$ apresentados nas Figuras 1, 2, 3 e 4 são considerados altos, o que indica um alto grau de ajuste das equações de regressão.

De modo geral, os peixes abatidos com pesos mais elevados proporcionaram maior RFSP e menores \%CAB e \%RF. Entretanto, a redução da \%CAB não foi suficiente para aumentar o RCAR devido, provavelmente, a um maior aumento da \%VIS, com o aumento do peso de abate.

Esses resultados foram semelhantes aos encontrados por Vilas Boas (2001), que estudando os rendimentos do processamento do matrinchã (Brycon cephalus, Günther, 1869), em diferentes classes de peso $\left(\mathrm{C}_{1}=750\right.$ g a $800 \mathrm{~g} ; \mathrm{C}_{2}=801 \mathrm{~g}$ a $850 \mathrm{~g} ; \mathrm{C}_{3}=851 \mathrm{~g}$ a $900 \mathrm{~g}$; e $\mathrm{C}_{4}=901 \mathrm{~g}$ a $950 \mathrm{~g}$ ), observou uma redução na porcentagem da cabeça, com o aumento da classe, sem haver influência significativa do peso de abate no rendimento de carcaça. Entretanto, ao contrário dos resultados obtidos no presente estudo, a porcentagem de vísceras diminuiu inicialmente em função da classe de peso, com posterior aumento; e os rendimentos de filé, com e sem pele, assim como a porcentagem de resíduo da filetação, não apresentaram influência significativa do peso em que os peixes foram abatidos.
Macedo-Viegas et al. (2000), estudando o efeito das classes de peso $\left(\mathrm{P}_{1}=400 \mathrm{~g}\right.$ a $500 \mathrm{~g}, \mathrm{P}_{2}=501 \mathrm{~g}$ a $600 \mathrm{~g} \mathrm{e}_{3}=601 \mathrm{~g}$ a $700 \mathrm{~g}$ ) sobre os rendimentos do processamento do matrinchã, não evidenciaram influência significativa do peso de abate nos rendimentos de filé, gordura visceral e resíduo. Segundo esses autores, os matrinchãs podem ser abatidos e processados com peso inferior ao normalmente comercializado, ou seja, na faixa entre $400 \mathrm{~g}$ a $700 \mathrm{~g}$, sem prejuízo do rendimento de filé, contrariando o observado neste estudo.

As porcentagens de filé e resíduos do surubim (Pseudoplatystoma coruscans, Agassiz, 1829), estudado por Ribeiro \& Miranda (1997), não apresentaram diferenças significativas entre as classes de peso $\left(\mathrm{C}_{1}=\right.$ 1.082 g a 5.000 g; $C_{2}=5.001$ g a 10.000 g; e $C_{3}=$ 10.000 g a $16.000 \mathrm{~g}$ ), ao contrário da porcentagem de vísceras, que aumentou acentuadamente (44\% entre as classes 1 e 3) com o crescimento. Nesse mesmo estudo, não se observou uma diferença prática das porcentagens da carcaça e da cabeça entre as classes de peso estudadas. Já o bagre-do-canal (Ictalurus punctatus, Rafinesque, 1818), estudado por Lovell \& Li (1992), apresentou uma diferença de $9 \%$ na porcentagem de carcaça entre indivíduos de $0,5 \mathrm{~g}$ e $1,5 \mathrm{~kg}$.

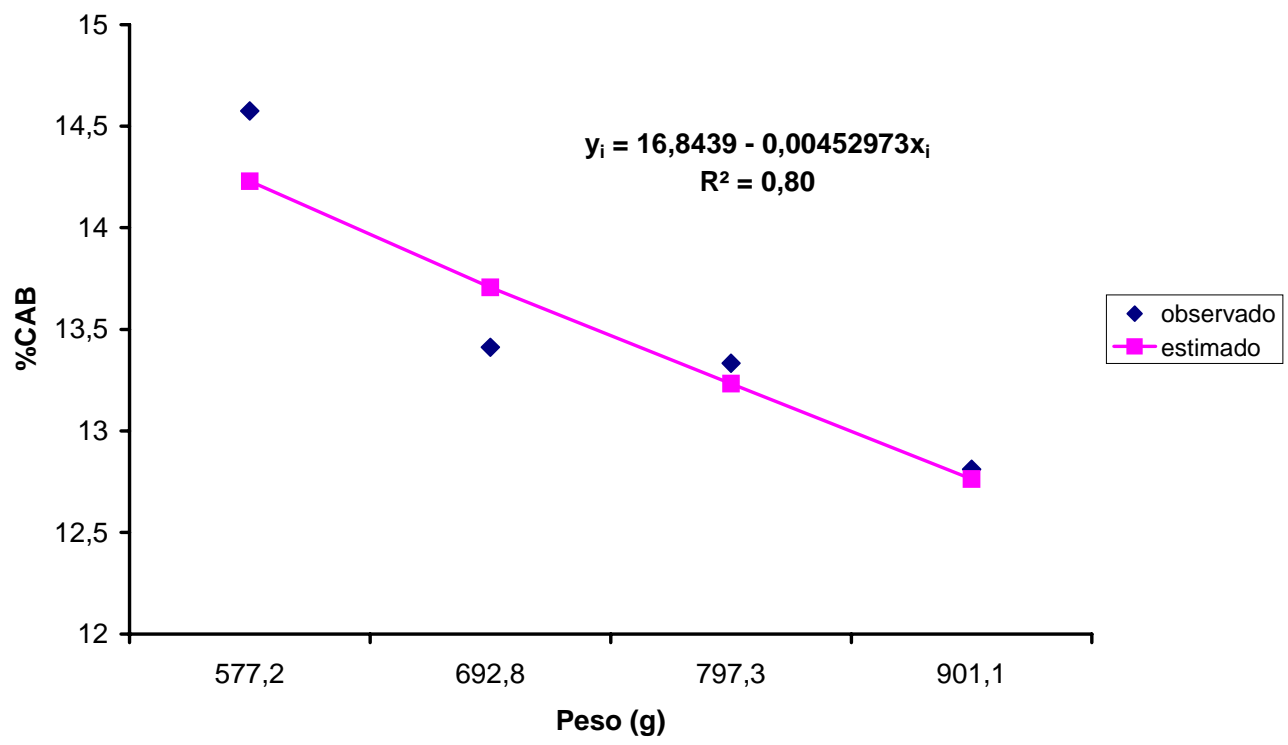

FIGURA 1 - Porcentagem da cabeça (\%CAB) da piracanjuba (Brycon orbignyanus), em função do peso de abate. 


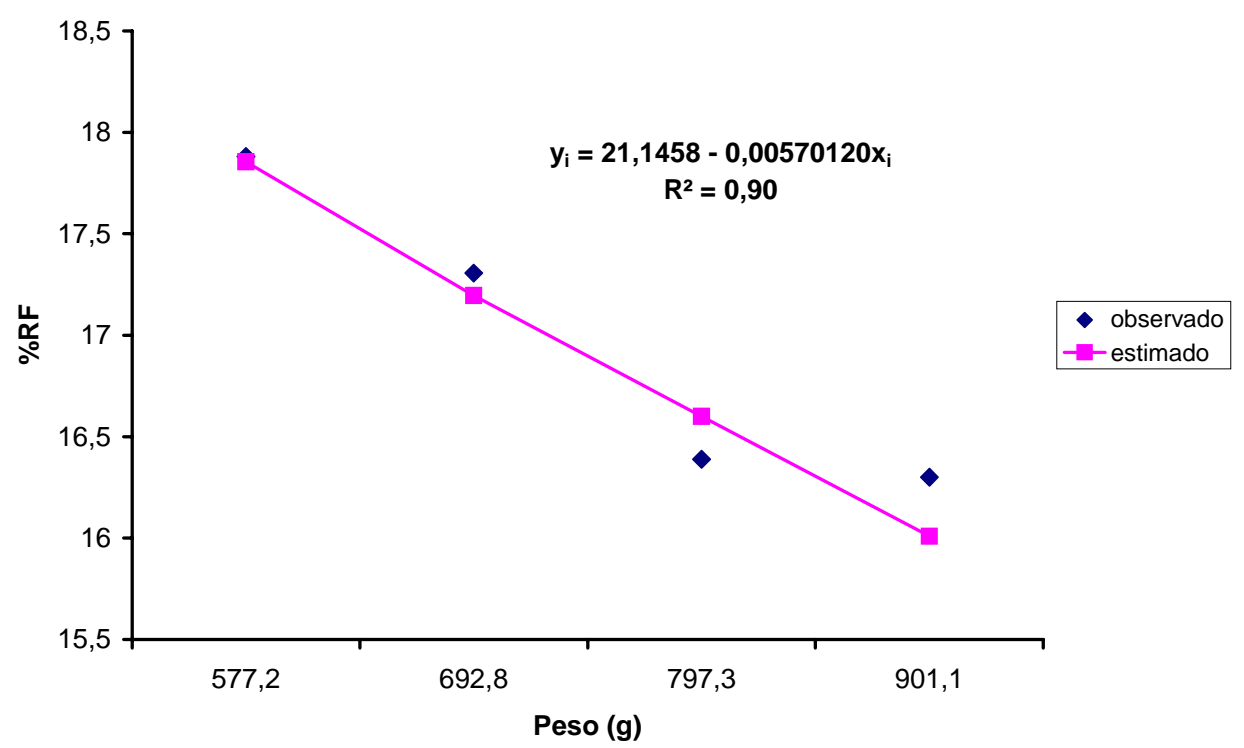

FIGURA 2 - Porcentagem do resíduo da filetação (\%RF) da piracanjuba (Brycon orbignyanus), em função do peso de abate.

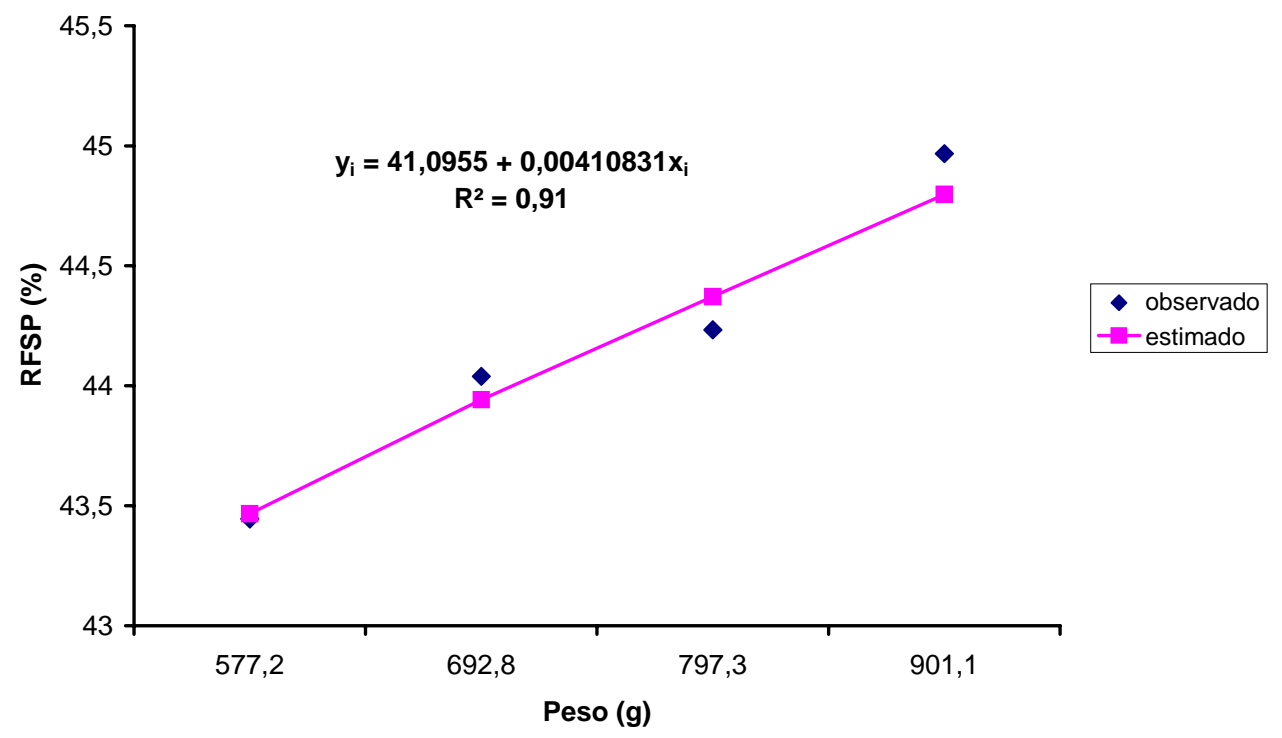

FIGURA 3 - Rendimento do filé sem pele (RFSP) da piracanjuba (Brycon orbignyanus), em função do peso de abate. 


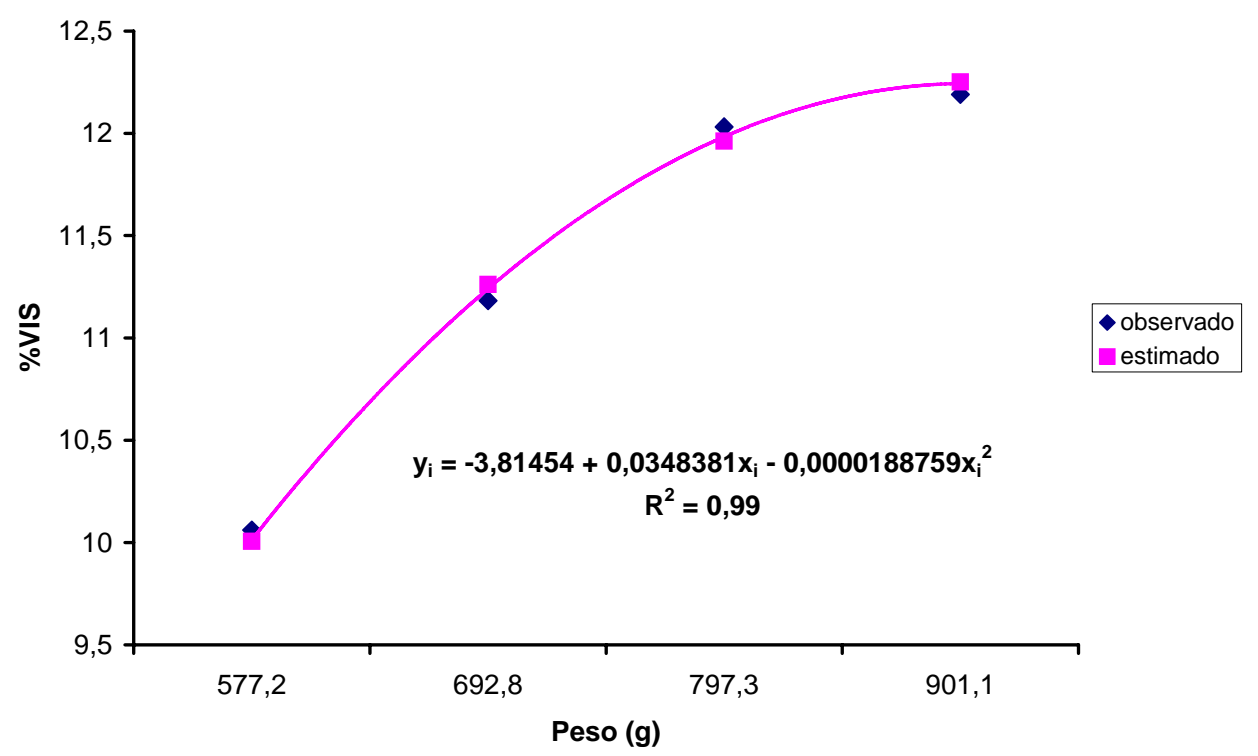

FIGURA 4 - Porcentagem de vísceras (\%VIS) da piracanjuba (Brycon orbignyanus), em função do peso de abate.

Estudando as características de carcaça de tilápias-do-nilo (Oreochromis niloticus, L.), em quatro classes de peso, Gonçalves et al. (2001) concluíram que os rendimentos de carcaça e filé não dependem do peso com que as tilápias-do-nilo são abatidas. Somente a quantidade de filé na carcaça é relacionada com esses pesos.

Portanto, ainda há muita controvérsia quanto à influência do peso de abate nos rendimentos do processamento dos peixes, sendo necessários mais estudos para a determinação do padrão de qualidade da carcaça e do peso ideal de abate para a piracanjuba e outras espécies cultiváveis de peixe, porém, com padronização nas metodologias de pesquisa nessa área.

\section{CONCLUSÕES}

Piracanjubas abatidas em classes de peso mais elevadas proporcionam maior rendimento de filé sem pele. Sendo assim, os peixes destinados ao processo de filetação devem ser abatidos, preferencialmente, com pesos mais elevados. Já os peixes cultivados com o intuito de serem comercializados na forma de carcaça ou inteiros eviscerados podem ser abatidos em pesos menores, pois a redução na porcentagem da cabeça não foi suficiente para aumentar o rendimento da carcaça, em razão, provavelmente, de um maior aumento da porcentagem de vísceras com o aumento do peso de abate.

\section{REFERÊNCIAS BIBLIOGRÁFICAS}

CONSELHO ESTADUAL DE POLÍTICA AMBIENTAL. Deliberação COPAM, n. 041/95. Belo Horizonte, v. 104, n. 14, p. 1-4, 1996.

CONTRERAS-GUZMÁN, E. S. Bioquímica de pescados e derivados. Jaboticabal: FUNEP, 1994. 409 p.

EYO, A. A. Carcass composition and filleting yield of ten fish species from Kainji Lake: proceedings of the FAO expert consultation on fish technology in Africa. FAO fishers Rport, Rome, n. 467, p. 173-175, 1993. Supplement.

FEIDEN, A.; HAYASHI, C. Desenvolvimento de alevinos de piracanjuba (Brycon orbignyanus Valenciennes, 1849), em tanques experimentais com diferentes adubações orgânicas. In: ENCONTRO ANUAL DA SOCIEDADE BRASILEIRA DE ZOOTECNIA, 36., 1999, Porto Alegre. Anais... Porto Alegre: UFRGS, 1999. p. 26-29.

GASPARINO, E.; CAMPOS, A. T.; KLOSOVKI, E. S.; GUERREIRO, P. K.; FULBER, V. M.; LEAL, D. M.; SOUSA, I. de. Estudos de parâmetros corporais em tilápia do Nilo (Oreochromis niloticus). In: AQÜICULTURA BRASIL 2002， 2002， Goiânia. Anais... Goiânia: ABRAq, 2002. p. 183. 
GONÇALVES, T. M.; ALMEIDA, A. J. L.; OLIVEIRA, H. N. Avaliação de características de carcaças de tilápias do Nilo (Oreochromis niloticus). In: REUNIÃO ANUAL DA SOCIEDADE BRASILEIRA DE ZOOTECNIA, 2001, Piracicaba. Anais... Piracicaba: SBZ, 2001. p. 38.

LOVELL, R. T.; LI, M. Comparison of feed conversion, dressing yield, and muscle composition for second and third-year channel catfish. Progressive Fish Culturist, Bethesda, v. 54, n. 3, p. 171-173, 1992.

MACEDO-VIEGAS, E. M.; SCORVO, C. M. D. F.; VIDOTTI, R. M.; SECCO, E. M. Efeitos das classes de peso sobre a composição corporal e o rendimento de processamento de matrinchã (Brycon cephalus). Acta Scientiarum, Maringá, v. 22, n. 3, p. 725-728, set. 2000 .

MACEDO-VIEGAS, E. M.; SOUZA, M. L. R.; KRONKA, S. N. Estudo da carcaça de tilápia do Nilo (Oreochonis niloticus), em quatro categorias de peso. Revista Unimar, Marília, v. 19 n. 3, p. 863-870, 1997.

RIBEIRO, L. P.; LIMA, L. C.; TURRA, E. M.; QUEIROZ, B. M.; RIBEIRO, T. G.; MIRANDA, M. O. T. Efeito do peso e do operador sobre o rendimento de filé em tilápia vermelha spp. In: AQÜICULTURA
BRASIL 98, 1998, Recife. Anais... Recife: ABRAq, 1998. v. 2, p. 773-778.

RIBEIRO, L. P.; MIRANDA, M. O. T. Rendimentos de processamento do surubim Psedoplatystoma coruscans. In: MIRANDA, M. O. T. (Org.). Surubim. Belo Horizonte: IBAMA, 1997. p. 101-111 (Coleção MeioAmbiente, Série Estudos Pesca, 19).

SOUZA, M. L. R.; LIMA, S.; FURUYA, W. M.; PINTO, A. A.; LOURES, B. T. R. R. Sex related effects on the processing yield of African catfish (Clarias gariepinus) In: AQÜICULTURA BRASIL 98, 1998, Recife. Anais... Recife: ABRAq, 1998. p. 321.

UNIVERSIDADE FEDERAL DE VIÇOSA. Sistemas de análises estatísticas e genéticas: versão 5.0. Viçosa, MG, 1992. Manual do usuário.

VAZ, M. M.; TORQUATO, V. C.; BARBOSA, N. D. C. Guia ilustrado de peixes da bacia do Rio Grande. Belo Horizonte: CEMIG/CETEC, 2000. 144 p.

VILAS BOAS, G. C. Morfometria, rendimento do processamento e composição química do filé de matrinchã Brycon cephalus (GÜNTHER, 1869). 2001. 59 p. Dissertação (Mestrado em Zootecnia) Universidade Federal de Lavras, Lavras, 2001. 